\title{
Oscillatory Mixed Differential Difference Equations
}

\author{
Sandra Pinelas \\ Universidade dos Açores \\ Departamento de Matemática \\ R. Mãe de Deus \\ 9500-321 Ponta Delgada \\ Portugal
}

Abstract: In this work is studied the oscillatory behavior of the delay differential difference equation of mixed type

$x^{\prime}(t)=\sum_{i=1}^{\ell} p_{i} x\left(t-r_{i}\right)+\sum_{j=1}^{m} q_{j} x\left(t+\tau_{j}\right) \quad\left(r_{i}>0, i=1, \ldots, \ell ; \tau_{j}>0, j=1, \ldots, m\right)$

Some criteria are obtained in order to guarantee that all solutions of (1) are oscillatory.

\section{Introduction}

The aim of this work is to study the oscillatory behavior of the differential difference equation of mixed type

$$
x^{\prime}(t)=\sum_{i=1}^{\ell} p_{i} x\left(t-r_{i}\right)+\sum_{j=1}^{m} q_{j} x\left(t+\tau_{j}\right)
$$

where $x(t) \in \mathbb{R}, 0<r_{1}<r_{2}<\ldots<r_{\ell}, 0<\tau_{1}<\tau_{2}<\ldots<\tau_{m}$ and the coefficients $p_{i}, q_{j}$ are real numbers.

As usual, we will say that a solution $x(t)$ of (??) oscillates if it has arbitrary large zeros. When all solutions oscillate (??) will be said oscillatory. 